\title{
EEG Contribution to the Diagnosis of Antibody-Negative Autoimmune Encephalitis: A Case Report
}

\author{
Mayusa Mito ${ }^{a} \quad K$ otaro Sakurai ${ }^{b} \quad$ Yuichi Nakamura $^{b} \quad$ Azusa Nagai ${ }^{c}$ \\ Sho Seo ${ }^{c}$ Keiko Tanaka $^{d}$ Ichiro Yabe ${ }^{c}$ Ichiro Kusumi ${ }^{b}$ \\ aDepartment of Psychiatry, Sapporo City General Hospital, Sapporo, Japan; bepartment of \\ Psychiatry, Hokkaido University Graduate School of Medicine, Sapporo, Japan; 'Department \\ of Neurology, Hokkaido University Graduate School of Medicine, Sapporo, Japan; \\ ${ }^{\mathrm{d} D e p a r t m e n t}$ of Animal Model Development, Brain Research Institute, Niigata University, \\ Niigata, Japan
}

Keywords

Autoimmune diseases - Diagnosis - Electroencephalography · Encephalitis - Seizures

\begin{abstract}
Autoimmune encephalitis $(\mathrm{AE})$ is a group of inflammatory brain diseases that are characterized by prominent neuropsychiatric symptoms. Early therapeutic intervention is important for AE. Therefore, without waiting for autoantibody test results, clinicians must consider the possibility of AE based solely on clinical symptoms and conventional test results. The case described herein is of antibody-negative encephalitis with abnormalities shown only by EEG, which contributed to the diagnosis and treatment. The patient, a 20-year-old woman, showed autonomic seizures in addition to movement disorders, psychiatric symptoms, and cognitive dysfunction, which worsened subacutely. Her seizures and movement disorders were not responsive to antiepileptic medications. Results obtained from MRI and cerebrospinal fluid (CSF) were normal; EEG findings showed repeated spikes in the right temporal area, with changes over time. Based on the clinical course and EEG, along with administered immunotherapy, which resolved seizures, movement disorders, and psychiatric symptoms, we suspected $A E$. For diagnosis of $A E$ and for evaluating treatment responsiveness, EEG was useful. Results indicate that EEG can assist clinicians even with AE cases for which MRI and CSF findings are normal.
\end{abstract}


Mito et al.: Usefulness of EEG in Autoimmune Encephalitis

\section{Introduction}

Autoimmune encephalitis (AE) constitutes a group of inflammatory brain diseases that are characterized by prominent neuropsychiatric symptoms. Common clinical features include changes in behavior, psychosis, seizures, memory and cognitive deficits, abnormal movements, dysautonomia, and a decreased level of consciousness [1]. In AE, early diagnosis and immunotherapy are important for a good outcome [2]. However, antibody tests are not readily available at many institutions. Several weeks are required to obtain the results. Furthermore, the possibility of immune-mediated diseases cannot be excluded even for a patient who is negative for autoantibodies. Therefore, the possibility of AE must be assessed during early stages of treatment and must be assessed based on clinical symptoms and conventional tests that are available to most clinicians. In recent years, practical, symptom-based diagnostic approaches have been proposed [3, 4]. Seizures are a common feature of AE. Nearly 70\% of AE show seizures. Nearly 85\% show EEG abnormalities, which are more common in younger patients [5]. Therefore, understanding seizure and EEG characteristics in AE is useful for supporting more rapid diagnosis and initiation of treatment. This report describes a case of antibody-negative encephalitis in which only EEG showed abnormalities. Results demonstrate that EEG was extremely useful for diagnosis and treatment.

\section{Case Report}

The patient was a 20-year-old right-handed Japanese woman. Her psychomotor development was normal. At the age of 20 years, she began to have sudden anxiety about once a week accompanied by autonomic symptoms such as palpitations, hyperventilation, and tears. In addition, cognitive decline, such as getting lost on familiar streets, memory loss, and somnolence also appeared. A few months later, anxiety attacks began to occur more than a few times a week. She also had a seizure by which the right side of her face twitched for several tens of seconds. Therefore, she visited a neurosurgery hospital. Brain MRI and MRA showed no abnormality. Subsequently, auditory hallucinations of "die," irritability, and personality change also appeared; she was referred to our hospital because of suspected mental disorder and epilepsy. Results of her neurological examination were normal. Scalp interictal EEG showed repetitive right posterior temporal spikes (Fig. 1a). Encephalitis, including autoimmune encephalitis, was suspected because the patient had seizures with autonomic symptoms, cognitive dysfunction, and psychotic symptoms, all of which progressed subacutely, and frequent temporal spikes on the EEG. However, no abnormal finding was found in relation to cerebrospinal fluid (CSF), which includes IgG index and oligoclonal bands. Therefore, we diagnosed her as having focal epilepsy with autonomic seizures: lacosamide (LCM) $100 \mathrm{mg} /$ day was initiated. Although LCM was increased to $250 \mathrm{mg} /$ day over the next 3 weeks, the seizures were not controlled. A few days later, she was brought to our hospital by ambulance because she had shown psychomotor agitation, screaming "die" and "kill me." After she lost consciousness, she had a transient respiratory arrest and involuntary movements such as licking her lips with her tongue. Because the disturbance of consciousness persisted for a long time, we diagnosed her as status epilepticus and administered diazepam, midazolam, and fosphenytoin intravenously. When she then regained consciousness, she was admitted. On the day following admission, EEG showed diffuse spike-waves intermittently, located with bilateral frontal dominance (Fig. 1b). Brain MRI and 18F-fluorodexyglucose-PET were normal. We increased LCM to $300 \mathrm{mg} /$ day. Then she was discharged. However, her autonomic seizures appeared after a week. We added levetiracetam (LEV), which was ineffective. In addition,

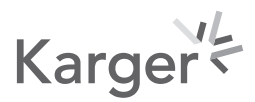




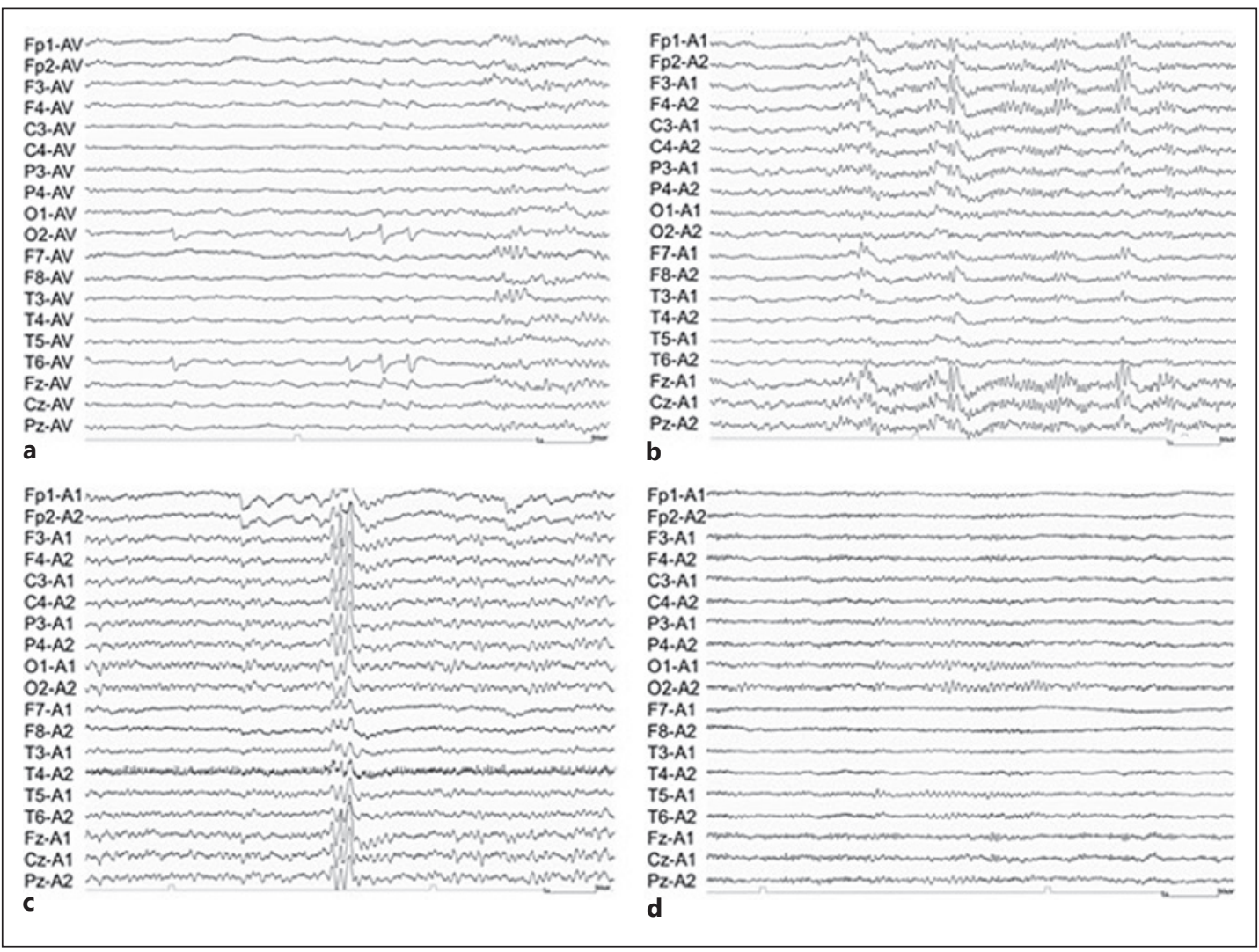

Fig. 1. Interictal EEG. a EEG at initial examination. EEG spikes frequently appear in the right posterior temporal area. b EEG at the first admission. Bilateral frontal dominant diffuse spike-waves appear intermittently. c EEG at second admission. Diffuse high-voltage 2-3 Hz slow waves appeared sporadically. d EEG after immunotherapy.

abnormal movements in the neck such as repeated swallowing and hemiballismus in the upper right limb appeared. The seizures and movement disorders occurred frequently. She was admitted to our hospital again. After admission, we increased LEV to 2,000 mg/day, and added perampanel in addition to LCM and LEV. Nevertheless, the symptoms were not controlled. Her irritability worsened. Furthermore, she began to complain of photophobia and weakness in her extremities. EEG showed continuous 5-6 $\mathrm{Hz} \theta$ waves, with sporadic diffuse high-voltage 2-3 Hz slow waves (Fig. 1c). No abnormality was found from the second CSF. We strongly suspected anti-N-methyl-D-aspartate receptor encephalitis. However, the antibody was negative. A whole-body MRI showed no tumor, including ovarian teratoma. Although no abnormality was found in her imaging or laboratory findings other than the EEG, we again strongly suspected autoimmune encephalitis because of the autonomic seizures and movement disorders, treatment resistance to antiepileptic drugs, cognitive dysfunction, and psychiatric symptoms. We started 2 courses of intravenous methylprednisolone therapy followed by 7 sessions of plasma exchange therapy under sedation and ventilator control. After administering these immunotherapies, the seizures and movement disorders improved markedly. Neurological symptoms such as irritability, weakness, and photophobia disappeared. Her mother said that she had reverted to her usual personality. After immunotherapy, diffuse high-voltage slow waves and $\theta$ waves on EEG had disappeared completely (Fig. 1d). However, when the oral prednisolone was tapered to $15 \mathrm{mg}$, autonomic seizures, anxiety that resembled panic attacks, and movement disorder (flapping of her limbs) appeared. EEG 
revealed frequently right posterior temporal spikes again. We administered the third course of intravenous methylprednisolone therapy. The therapy was successful. She was discharged on $15 \mathrm{mg}$ of oral prednisolone. Although her serum and CSF were sent to other institutions for screening for typical autoantibodies that can be measured in Japan at present, all results were negative: NMDAR, LGI1, CASPR2, AMPAR, GABAbR, GlyR, and AQP4. Paraneoplastic neurosyndrome-related antibodies were also negative: AMPH, CV2, PNMA2, Ri, Yo, Hu, recovern, SOX1, titin, Zic4, GAD65, and Tr.

\section{Discussion}

This report describes a case of antibody-negative AE in which repeated EEG measurements were very useful for diagnosis and evaluation of therapeutic response. Although frequently reported, AE-related autoantibodies were negatively indicated, and although lack of abnormality was found from clinical examinations (CSF, MRI, and fluorodexyglucose-PET) other than EEG, we diagnosed the patient as having AE. Moreover, she showed epileptic seizures that were accompanied by autonomic symptoms, and which were refractory to antiepileptic, in addition to movement disorders, psychiatric symptoms, and cognitive dysfunction that worsened subacutely. She also met the diagnostic criteria for possible autoimmune encephalitis by Glaus et al. [3]. Furthermore, her good response to immunotherapy supports the AE diagnosis. For AE, MRI and basic CSF often show normal findings, as in our patient [6]. In such case, EEG might be a more sensitive tool for diagnosis. Recent systematic reviews found that nearly $85 \%$ of patients with AE had some abnormality on EEG [5]. The most common EEG abnormality was slow waves (51.1\%). Epileptiform discharges appeared in $21.6 \%$ of the patients [5]. Reports of some other studies have described that when epileptic discharges appear in EEG, they are most frequently distributed over the temporal region [7, 8]. Furthermore, it is noteworthy that in AE, changes in seizure semiology and EEG findings over time have often been seen [7]. In our case, EEG changed one after another, from right posterior temporal spikes (Fig. 1a) to frontal dominant diffuse spike-waves (Fig. 1b) and further to diffuse high-voltage 2-3 Hz slow waves (Fig. 1c). Subacute changes in the seizure type or EEG findings are uncommon in epilepsy syndromes because of structural or genetic etiologies. Therefore, one should strongly suspect the possibility of immune etiology in such cases. In our patient, the EEG abnormality disappeared after immunotherapy and reappeared with the relapse of symptoms (Fig. 1d). Results indicate that repeated EEG measurements are very useful not only for diagnosis but also for assessing disease activity and treatment response. Although we measured conventional EEG repeatedly, we were unable to record the ictal EEG because of the lack of continuous EEG monitoring. For AE, subclinical seizures (SCs) are reportedly observed occasionally on continuous EEG monitoring in addition to clinical seizures (CSs) [7, 8]. Furthermore, comparison of the relative proportions of CSs and SCs between antibody-positive and negative AE showed that the proportion of SCs was significantly higher in the antibody-negative cases [8]. We infer that continuous EEG monitoring and recording not only of CSs but also of SCs would have provided more important information for the diagnosis of AE.

\section{Conclusion}

We described a case of antibody-negative AE for which repeated EEG measurements were very useful for the diagnosis and for the evaluation of therapeutic response. Results show that EEG can assist clinicians even in AE cases with normal MRI and CSF findings.

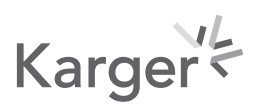




\section{Statement of Ethics}

This research was conducted ethically in accordance with the World Medical Association Declaration of Helsinki. The patient described in this paper gave written informed consent to publication of the case, including publication of images. The paper is exempt from ethical committee approval because it is a case report for which consent was obtained from the patient.

\section{Conflict of Interest Statement}

The authors have no conflict of interest to report.

\section{Funding Sources}

No author has received any funding for the conduct, authorship or publication of this study.

\section{Author Contributions}

M. Mito contributed to medical treatment, data acquisition, drafting, and writing. K. Sakurai contributed to medical treatment, data acquisition, visualization, and revision of the manuscript. Y. Nakamura contributed to revision of the manuscript. A. Nagai and S. Seo contributed to medical treatment. K. Tanaka contributed to data acquisition. I. Yabe and I. Kusumi contributed to supervision. All authors reviewed and approved the final draft.

\section{Data Availability Statement}

All data generated or analyzed during this study are included in this article. Further inquiries can be directed to the corresponding author.

\section{References}

1 Dalmau J, Graus F. Antibody-mediated encephalitis. N Engl J Med. 2018 Mar 1;378(9):840-51.

2 Byrne S, Walsh C, Hacohen Y, Muscal E, Jankovic J, Stocco A, et al. Earlier treatment of NMDAR antibody encephalitis in children results in a better outcome. Neurol Neuroimmunol Neuroinflamm. 2015 Aug;2(4): e130.

3 Graus F, Titulaer MJ, Balu R, Benseler S, Bien CG, Cellucci T, et al. A clinical approach to diagnosis of autoimmune encephalitis. Lancet Neurol. 2016 Apr;15(4):391-404.

4 Dubey D, Alqallaf A, Hays R, Freeman M, Chen K, Ding K, et al. Neurological autoantibody prevalence in epilepsy of unknown etiology. JAMA Neurol. 2017 Apr 1;74(4):397-402.

5 Yeshokumar AK, Coughlin A, Fastman J, Psaila K, Harmon M, Randell T, et al. Seizures in autoimmune encephalitis - a systematic review and quantitative synthesis. Epilepsia. 2021 Feb;62(2):397-407.

6 Hermetter C, Fazekas F, Hochmeister S. Systematic review: syndromes, early diagnosis, and treatment in autoimmune encephalitis. Front Neurol. 2018;9:706.

7 Steriade C, Moosa ANV, Hantus S, Prayson RA, Alexopoulos A, Rae-Grant A, et al. Electroclinical features of seizures associated with autoimmune encephalitis. Seizure. 2018 Aug;60:198-204.

8 Morano A, Fanella M, Cerulli Irelli E, Barone FA, Fisco G, Orlando B, et al. Seizures in autoimmune encephalitis: findings from an EEG pooled analysis. Seizure. 2020 Dec;83:160-8.

\section{Karger'}

